\title{
Asociación de la hemorragia prerretiniana con la presencia y la gravedad de la retinopatía del prematuro en pacientes de alto riesgo
}

\author{
Daniel Garza-Cantú, Saria Sánchez-Álvarez, David Ramón Palacios-Barragán \\ y Gerardo del Carmen Palacios-Saucedo \\ Departamento de Oftalmología y División de Investigación, Unidad Médica de Alta Especialidad No. 25, Instituto Mexicano del Seguro Social, \\ Monterrey, N.L., México
}

\section{Resumen}

Objetivo: Evaluar si la hemorragia prerretiniana (HPR) se asocia a la presencia y la gravedad de la retinopatía del prematuro $(R O P)$ en pacientes de alto riesgo. Método: Estudio de cohortes prospectivo en el que se incluyeron pacientes referidos al Departamento de Oftalmología para tamizaje de ROP durante octubre y noviembre de 2016, los cuales fueron evaluados semanalmente en cuatro ocasiones para valorar la relación con HPR y el desarrollo de ROP, así como su gravedad. Se utilizaron frecuencias absolutas, medianas con valores mínimo y máximo, prueba de ji al cuadrado y prueba U de Mann-Whitney, así como riesgo relativo con intervalo de confianza del 95\%. Resultados: De un total de 30 pacientes, en la primera semana 11 femeninas (36\%) y 4 masculinos (13\%) presentaron HPR; en la segunda semana, 13 (43\%) presentaron HPR y 14 (46\%) desarrollaron ROP; en la tercera semana no hubo cambios; en la última semana, 8 presentaron HPR (26\%) y 11 (36\%) ROP. El estadio I de gravedad se observó con mayor frecuencia. Se encontró significancia estadística $(p=0.040)$ en la presencia de HPR y el desarrollo de ROP en la primera semana. Conclusiones: La HPR es un factor de riesgo para el desarrollo de ROP durante las primeras semanas de vida y se asocia a estadios de gravedad leve.

PALABRAS CLAVE: Hemorragia prerretiniana. Retinopatía del prematuro. Gravedad.

\begin{abstract}
Objective: To assess whether preretinal hemorrhage $(P R H)$ is associated with the presence and severity of retinopathy of prematurity (ROP) in high-risk patients. Methods: Prospective cohort study, patients referred to the Department of Ophthalmology for ROP screening during October-November 2016 were evaluated weekly on 4 occasions to assess the relationship with $\mathrm{PRH}$ and the development of ROP as well as degree of severity associated. We used absolute, median frequencies with minimum and maximum values, $\chi^{2}$ test and Mann-Whitney U-test, as well as relative risk with $95 \%$ confidence interval. Results: $A$ total of 30 patients, in the first week 11 females (36\%) and 4 males (13\%) had PRH; in the $2^{\text {nd }}$ week 13 patients (43\%) presented $\mathrm{PRH}$ and 14 (46\%) developed ROP; on the $3^{\text {rd }}$ week there were no changes; in the last week 8 presented HPR (26\%) and 11 patients (36\%) with ROP. Stage I severity occurred more frequently. Statistical significance $(p=0.040)$ was found in the presence of PRH and the development of ROP in the first week with. Conclusions: HPR is a risk factor for the development of ROP during the first weeks of life and is associated with stages of mild severity.
\end{abstract}

KEY WORDS: Preretinal hemorrhage. Retinopathy of prematurity. Severity.

\author{
Correspondencia: \\ Saria Sánchez-Álvarez \\ Laguna de Magdalena, 7511 \\ Col. Versalles \\ C.P. 32420, Cd. Juárez, Chih., México \\ E-mail: shiver_16@ hotmail.com
}

Fecha de recepción: 15-12-2016

Fecha de aceptación: 11-01-2017

DOI://dx.doi.org/10.24875/GMM.17003107
Gac Med Mex. 2017;153:818-823

Contents available at PubMed www.gacetamedicademexico.com 


\section{Introducción}

La retinopatía del prematuro $(\mathrm{ROP})$ se define como la alteración vasoproliferativa de la retina progresiva que se presenta exclusivamente en infantes prematuros $^{1}$. En todo el mundo se reportan incidencias que van del 12 al $78 \%$, en cualquiera de sus estadios, en niños nacidos con menos de $1500 \mathrm{~g}$ de peso-4.

Dentro de los factores sistémicos asociados se encuentran la inmadurez vascular, el estado de hipoxia, la edad gestacional, el embarazo múltiple, la dificultad respiratoria, la apnea, la acidosis, la bradicardia, la anemia, la sepsis y las transfusiones ${ }^{5,6}$.

La ROP cuenta con una clasificación internacional que considera tres zonas concéntricas que describen la retina desde su porción posterior a la anterior (zona), extensión circunferencial (zona horaria o meridianos) y gravedad (etapa) con presencia o ausencia de enfermedad «plus»:

- Zona I: con el disco óptico como centro, y el doble de distancia desde la papila a la fóvea, el círculo formado es la zona I.

- Zona II: inicia en el borde de la zona I y se extiende hacia la ora serrata nasalmente con el área temporal correspondiente $\mathrm{o}$ al ecuador.

- Zona III: inicia en el borde de la zona II y es el área de retina restante hacia el sector temporal. Es la última zona en completar la vascularización para el desarrollo completo de la circulación retiniana.

La extensión de la ROP se documenta de acuerdo con el número de zonas horarias involucradas. Existen cinco estadios:

- Estadio I o línea de demarcación: se observa una línea de demarcación entre las áreas avascular y vascular de la retina.

- Estadio II o cresta: la línea de demarcación crece hasta ocupar un volumen y tiene altura y anchura para formar una cresta por encima del plano de la retina. Se pueden observar también pequeños copos o penachos de vasos sanguíneos, llamados ordinariamente popcorn, localizados posterior a la cresta.

- Estadio III o proliferación fibrovascular: se observa tejido fibrovascular extrarretiniano que se extiende desde la cresta hasta el vítreo; puede ser o no continuo y se localiza posterior a la cresta.

- Estadio IV o desprendimiento de retina subtotal o parcial: se observa desprendimiento de retina parcial de tipo exudativo o traccional. Se divide en $A$, cuando no involucra la mácula, y $B$ cuando existe involucro de la misma.

- Estadio V o desprendimiento de retina total: se observa desprendimiento de retina total, siendo evidente clínicamente leucocoria.

Existen además variantes de gravedad, como la «enfermedad plus», que se define como dilatación venosa y tortuosidad arterial de la vasculatura del polo posterior. La enfermedad «pre-plus» es también una alteración en la vasculatura manifestada por dilatación y tortuosidad mayor de lo normal, pero de menor intensidad que la enfermedad plus.

Se ha descrito también la ROP posterior agresiva (anteriormente conocida como «enfermedad rush»), forma rara de presentación rápidamente progresiva que se observa en el polo posterior como enfermedad plus grave y neovascularización intrarretiniana plana, que puede progresar fácilmente a estadio IV y provocar ceguera total si no es tratada de forma temprana?. La técnica de exploración debe realizarse bajo dilatación pupilar farmacológica y oftalmoscopia indirecta con uso de lente de 28 dioptrías. Generalmente se utilizan soluciones oftálmicas para dilatar a base de ciclopentolato $(0.5 \%)$ y fenilefrina $(2.5 \%)$, las cuales se aplican cada 15 minutos durante tres veces ${ }^{2,7} . \mathrm{La}$ Academia Americana de Pediatría establece que los infantes con peso al nacer de $1500 \mathrm{~g}$ o edad gestacional menor de 30 semanas de gestación, y ciertos casos particulares con peso al nacer de $1500-2000 \mathrm{~g}$ o edad gestacional mayor de 30 semanas de gestación con un estado clínico inestable, deben ser evaluados en búsqueda de ROP. El tamizaje debe realizarse 4 semanas posteriores al nacimiento ${ }^{7}$. Una vez estadificada la ROP se deben considerar los diferentes tratamientos que existen. La crioterapia consiste en el uso de una proba para congelar las áreas de retina avascular y evitar o disminuir el riesgo de pliegues o desprendimientos de retina secundarios a la retinopatía; sin embargo, debido al alto riesgo de complicaciones, como inflamación, y a la necesidad de anestesia general en el paciente pediátrico, hoy ya no es considerada como la primera opción de tratamiento ${ }^{8}$. La fotocoagulación indirecta con láser ha demostrado ser el tratamiento de primera línea (gold standard). Consiste en un sistema indirecto de luz infrarroja que provoca la ablación de la retina hipóxica convirtiéndola en retina anóxica, con la consiguiente reducción del estímulo vasogénico, la formación de vasos anormales y la progresión de la enfermedad ${ }^{9}$. Los fármacos que bloquean el factor de crecimiento 
endotelial o anti-VEGF (anti-vascular endotelial growth factor) son tratamientos que se aplican en inyección intravítrea $^{10}$. El tratamiento quirúrgico se encuentra reservado para casos de enfermedad muy avanzada, como los estadios 4 y 5 . Se ha demostrado que los mejores resultados se presentan en el estadio $4^{a}$, y dentro de los procedimientos que se pueden ofrecer están la vitrectomía y el cerclaje. En los casos en estadio 5 se puede considerar una vitrectomía por lensectomía o a cielo abierto; sin embargo, a partir del estadio 4B existe un alto riesgo de pérdida visual total posterior al tratamiento quirúrgico, por lo que se vuelve debatible la elección de cirugía o el seguimiento y la observación del paciente ${ }^{11}$.

La ROP es una patología generalmente autolimitada, con una duración promedio de 15 semanas, comprendidas desde el inicio de la enfermedad hasta el periodo de inactivación de la misma ${ }^{12}$. Finalmente, dentro de los factores de riesgo que se encuentran estrechamente relacionados con el desarrollo de complicaciones, y que además forman parte del objetivo de realizar este estudio, está la presencia de hemorragia vítrea y en particular la hemorragia prerretiniana (HPR). La hemorragia retiniana no es un hallazgo poco común en la ROP; se ha reportado con una incidencia que va del 5 al $33 \%$ en los pacientes con algún estadio de $\mathrm{ROP}^{13}$. El objetivo de este estudio consistió en evaluar si la HPR se asocia a la presencia y la gravedad de la ROP en pacientes de alto riesgo.

\section{Método}

El estudio se llevó a cabo en pacientes lactantes prematuros vistos en el Departamento de Oftalmología de la Unidad Médica de Alta Especialidad (UMAE) No. 25 del Instituto Mexicano del Seguro Social, en Monterrey, Nuevo León, para tamizaje de ROP durante octubre y noviembre de 2016. Se incluyeron pacientes prematuros de menos de 30 semanas de gestacion al nacimiento y mas de 30 días de nacidos al momento de la valoracion, con un peso bajo para la edad gestacional menor de $1500 \mathrm{~g}$, y con factores de riesgo para ROP, como uso de terapia con oxígeno. Se sometió a la evaluacion del comité de etica de la unidad médica y se obtuvo su autorización, además de realizarse un consentimiento informado para paciente pediatrico autorizado y firmado por el tutor de cada paciente. Se realizaron cuatro valoraciones semanales para exploración de fondo de ojo bajo dilatación farmacológica utilizando tropicamida $(0.8 \%)$ y fenilefrina $(0.5 \%)$, aplicadas cada 15 minutos durante tres veces. Se obtuvieron dos grupos: un primer grupo de pacientes que presentó HPR sin ROP y un segundo grupo sin HPR ni ROP en la primera evaluación. Se observaron y registraron los hallazgos obtenidos en las semanas segunda, tercera y cuarta, comparando la presencia de HPR, ROP y gravedad, además de los tratamientos indicados en los casos necesarios. Para el análisis estadístico de los resultados se midieron frecuencias absolutas y porcentajes, así como medias o medianas; para variables cualitativas se utilizó la prueba de ji al cuadrado, para muestras independientes las pruebas $t$ de Student y U de Mann-Whitney, y para la magnitud de la asociación se midió el riesgo relativo con su intervalo de confianza del $95 \%$. Se consideró como significativo un valor de $p<0.05$. Se empleó el paquete estadístico SPSS versión 17.0.

\section{Resultados}

Se realizó en la UMAE No. 25 de Monterrey durante los meses de octubre y noviembre de 2016. Se incluyeron 30 pacientes prematuros que acudieron a valoración para tamizaje de ROP, de los cuales 21 fueron niñas (70\%) y 9 hombres (30\%); además, el $36 \%$ de las femeninas y el $13 \%$ de los varones presentaron HPR, con un valor de $p=0.028$ y una relación estadísticamente significativa entre la presencia de HPR y el sexo del paciente. La mediana de edad al momento de la valoración fue de 33 días de vida (rango: 30-39), la edad gestacional promedio fue de 28 semanas (rango: 27-30), el peso promedio al nacer fue de $1067 \mathrm{~g}$ (rango: 645-1725) y el tiempo promedio de oxigenoterapia fue de 27 días (rango: 14-35) (Tabla 1).

En la segunda valoración se observó una disminución en la incidencia de HPR de hasta el $43.3 \%$. También fue evidente el desarrollo de ROP en 23 pacientes $(76.7 \%)$, obteniendo un valor de $p=0.04$ con significancia estadística. Además, 19 pacientes (63.3\%) presentaron estadio I de gravedad y 4 pacientes $(13.3 \%)$ estadio II. En la tercera revisión, el porcentaje de HPR no cambió y la presentación de ROP se manifestó en 25 pacientes (83.3\%), con $p=0.165$, sin modificarse el porcentaje de presentación del estadio I de gravedad, aumentado a 5 pacientes (16.7\%) con estadio II y 1 paciente (3.3\%) con estadio III, que supuso la mayor gravedad observada durante el estudio. Por último, en la cuarta semana se observó HPR en 8 pacientes (26.7\%), con un valor de $p=0.018$, demostrando una disminución y la resolución de la 
Tabla 1. Características clínicas de 30 pacientes lactantes prematuros clasificados según la presencia de HPR en la primera valoración del estudio

\begin{tabular}{|c|c|c|c|c|}
\hline & \multirow{2}{*}{$\begin{array}{c}\text { Total } \\
(n=30)\end{array}$} & \multicolumn{2}{|c|}{ HPR } & \multirow[t]{2}{*}{$p$} \\
\hline & & Sí (n = 15) & No $(n=15)$ & \\
\hline \multicolumn{5}{|l|}{ Sexo } \\
\hline Masculino & $9(30 \%)$ & $4(13 \%)$ & $5(16 \%)$ & 0.028 \\
\hline Femenino & $21(70 \%)$ & $11(36 \%)$ & $10(33 \%)$ & \\
\hline Edad (días) & $33(30-39)$ & $34(30-38)$ & $33(31-39)$ & 0.539 \\
\hline Edad gestacional (semanas) & $28(27-30)$ & $28(27-30)$ & $29(27-30)$ & 0.967 \\
\hline Peso al nacer (g) & $1067(645-1725)$ & $945(645-1725)$ & $1100(910-1425)$ & 0.098 \\
\hline Oxigenoterapia (días) & $27(14-35)$ & $23(14-35)$ & $28(21-35)$ & 0.081 \\
\hline
\end{tabular}

Los valores se presentan en frecuencias absolutas (porcentajes) y mediana (mínimo-máximo).

HPR: hemorragia prerretiniana.

Tabla 2. Asociación de la HPR en la primera semana del estudio con la presencia de ROP en las siguientes 3 semanas en 30 pacientes lactantes prematuros

\begin{tabular}{|c|c|c|c|c|c|}
\hline & \multirow{2}{*}{$\begin{array}{c}\text { Total } \\
(n=30)\end{array}$} & \multicolumn{2}{|c|}{ HPR en la primera semana } & \multirow[t]{2}{*}{ RR (IC 95\%) } & \multirow[t]{2}{*}{$\mathrm{p}$} \\
\hline & & Sí (n = 15) & No $(n=15)$ & & \\
\hline \multicolumn{6}{|l|}{ ROP } \\
\hline Semana 2 & $23(76 \%)$ & $14(46 \%)$ & $9(30 \%)$ & $1.55(1.00-2.40)$ & 0.040 \\
\hline Semana 3 & $25(83 \%)$ & $14(46 \%)$ & $11(36 \%)$ & $1.27(0.91-1.77)$ & 0.165 \\
\hline Semana 4 & $17(56 \%)$ & $11(36 \%)$ & $6(20 \%)$ & $1.83(0.91-3.65)$ & 0.070 \\
\hline
\end{tabular}

Los valores se presentan en frecuencias absolutas (porcentajes), mediana (mínimo-máximo), riesgo relativo con IC $95 \%$ y valor de $P$ calculado con la prueba de ji al cuadrado.

HPR: hemorragia prerretiniana; IC 95\%: intervalo de confianza del 95\%; ROP: retinopatía del prematuro; RR: riesgo relativo.

misma en un número de casos considerable en comparación con el inicio del estudio. Diecisiete pacientes (56.7\%) continuaron con ROP, con predominio del estadio I en 11 (36.7\%) de ellos, 9 pacientes (30\%) sin ROP con retina avascular y notoriamente 4 (13.3\%) sin ROP con retina vascularizada y completamente madura (Tabla 2).

Se analizó el riesgo relativo en relación con la presencia de HPR y desarrollo de ROP. En la segunda semana, el riesgo relativo de desarrollar ROP habiendo presentado HPR fue de 1.55 (1.0-2.4), representado un resultado significativo. Finalmente, en la cuarta semana, el riesgo relativo de presentar HPR fue de 1.83 (0.91-3.65) (Tabla 2).

Los pacientes que presentaron ROP en estadio I y II se valoraron semanalmente sin recibir tratamiento médico ni quirúrgico. Aquellos en estadio III requirieron manejo médico con antiangiogénicos intravítreos y seguimiento semanal estrecho. Los pacientes con retina avascular sin ROP fueron valorados cada 15 días, y aquellos con retina vascularizada se dieron de alta. Durante el estudio no se presentaron pacientes en estadios IV o V, por lo que no fue necesario el tratamiento con fotocoagulación, crioterapia ni cirugía.

\section{Discusión}

La ROP es una enfermedad exclusiva del infante prematuro que se manifiesta por una alteración vasoproliferativa progresiva que generalmente se resuelve por completo; sin embargo, existen casos graves que presentan secuelas importantes que comprometen la función y el pronóstico visual del paciente, como la hemorragia vítrea, el desprendimiento de retina, el glaucoma neovascular y la ptisis bulbi, los cuales culminan en una limitación visual importante o ceguera $\mathrm{A}^{7,8}$.

La ROP se presenta con mayor prevalencia en los países en vías de desarrollo, constituyendo un problema de salud pública importante. Presenta una incidencia mundial variable, de un 12 a un $78 \%$ de los recién nacidos que cumplen criterios de tamizaje ${ }^{4}$. La importancia del tamizaje se ve reflejada en el diagnóstico y el tratamiento oportuno, sobre todo en aquellos infantes que presentan estadios iniciales y que aún no han desarrollado complicaciones. Esta prueba de cribado se debe realizar en todo paciente que haya cumplido al menos 30 días de vida extrauterina, con edad gestacional menor de 30 semanas y peso al nacer menor de $1500 \mathrm{~g}^{7}$. La edad gestacional menor 
de 30 semanas, el peso al nacer menor de $1500 \mathrm{~g} \mathrm{y}$ la oxigenoterapia son los factores principalmente asociados a la presentación de esta patología ${ }^{7,8}$.

El objetivo principal de este estudio fue comparar la presencia de HPR en los pacientes valorados y la relación que existe con el desarrollo de ROP y su gravedad. La hemorragia vítrea ha sido reconocida como un factor de riesgo inicial para el desarrollo y la progresión de la ROP desde 1913 por Oguchi' ${ }^{14}$. Se han realizado diferentes estudios para explicar la fisiopatología de la hemorragia vítrea en la evolución natural de la ROP, como el de Cleary y Ryan ${ }^{15}$, quienes demostraron el papel de la sangre intraocular en el desarrollo de retinopatía proliferativa posterior a cirugía penetrante. Ehrenberg, et al. ${ }^{16}$ confirmaron que los cambios patológicos ocurrían incluso sin antecedente de cirugía penetrante. Hutcheson, et al..$^{15}$ reportaron en su estudio una incidencia de solo el $3 \%$ en los infantes valorados, pero que sin embargo progresaron a un estadio de enfermedad límite de alto riesgo. A la fecha no se han reportado estudios que comparen la presencia de HPR como factor de riesgo asociado al desarrollo de ROP, motivo por el cual se decidió realizar el presente trabajo. Dentro de los resultados obtenidos en este estudio, se observó que la incidencia de HPR presentó valores del $43.3 \%$ en las primeras revisiones y se redujo hasta el $26.7 \%$ en la cuarta semana; aun así, presentó un valor estadísticamente significativo, contrastando con los resultados obtenidos por Hutchesony la hemorragia vítrea. La incidencia de ROP fue del $76.6 \%$ en la segunda semana, considerándose un valor estadísticamente significativo, con variaciones a lo largo de las semanas siguientes y presentando un valor final de un $56.7 \%$ de incidencia, con resultados estadísticamente no significativos. Por tanto, la presencia de HPR constituye un factor de riesgo importante para la aparición y el desarrollo de ROP en las fases iniciales de la enfermedad.

Otro de los objetivos fue investigar la asociación que existe entre la presencia de ROP y su gravedad. Se observó que con mayor frecuencia se presenta el estadio I, con un valor que va del $63.3 \%$ inicial hasta el $36.7 \%$ en la cuarta semana, seguido de la presencia de retina avascular sin datos de ROP en el $23.3 \%$ hasta el $30 \%$ del total de los pacientes valorados al final de las 4 semanas, destacando la presentación del estadio III como el de mayor gravedad, en el $13.3 \%$ de los casos. Por otra parte, en el $13.3 \%$ de los pacientes hubo una progresión favorable hasta observarse la retina libre de alteraciones y con vascularización completa, lográndose egresar del programa de tamizaje sin mayor complicación. Existe diferencia considerable con los resultados obtenidos por Hutcheson, et al. en su estudio, donde la mayoría de los pacientes con hemorragia vítrea evolucionan a estadio IV avanzado y posteriormente a desprendimiento de retina.

Actualmente, los criterios para el tratamiento de la ROP están bien establecidos y fueron valorados en el presente estudio ${ }^{8}$. A lo largo del trabajo se realizó una valoración semanal para determinar los hallazgos en la exploración del fondo de ojo, así como el tratamiento indicado, en caso de necesitarlo. Se encontró que, al inicio del estudio, el $100 \%$ de los infantes presentaron en la exploración del fondo de ojo una retina inmadura y avascular; la edad promedio de escrutinio fue de 33 días de vida. En la segunda semana ocurre un cambio importante al manifestarse ROP en el $76.7 \%$, que se estadificaron como estadios I y II, sin requerir tratamiento. En la tercera y la cuarta semanas se hace evidente la progresión en cuatro pacientes, los cuales progresan a estadio III y resulta inminente iniciar tratamiento médico mediante la aplicación de antiangiogénicos intravítreos, marcando un seguimiento estrecho cada semana. Como ya se ha mencionado, Hutcheson, et al. reportaron que la mayoría de sus pacientes con hemorragia vítrea y ROP progresaron a estadio IV con desprendimiento de retina, siendo manejados con vitrectomía. Finalmente, cabe mencionar que al final del estudio cuatro pacientes fueron egresados del programa tras demostrar una respuesta favorable al observarse vascularización de la retina, sin necesidad de mayor seguimiento posterior, demostrando que esta patología generalmente se resuelve en semanas, con un promedio de 16 semanas posteriores al diagnóstico.

\section{Conclusiones}

La presencia de HPR se asocia de manera significativa con el desarrollo de ROP y resulta un factor de riesgo independiente para la predicción de la enfermedad en las primeras semanas de vida; posteriormente se desarrolla aun sin la presencia de HPR, muy probablemente a causa de otros factores.

La gravedad de la ROP se manifiesta con estadios leves en la mayoría de los casos, sin necesidad de tratamiento médico ni intervención quirúrgica, con seguimientos semanales, representando un factor de pronóstico visual favorable para el paciente. 
Se confirma que la evolución natural de la ROP se resuelve en las semanas posteriores al diagnóstico y de forma favorable en la mayoría de los casos. Además, se hace evidente la importancia de continuar realizando el tamizaje a la edad establecida por las guías internacionales de ROP para disminuir el riesgo de progresión, poder hacer un diagnóstico y un tratamiento oportuno, y así evitar complicaciones que afecten el pronóstico visual del paciente, así como las implicaciones personales y socioeconómicas para el paciente en un futuro.

\section{Financiamiento}

Los autores declaran no haber recibido financiamiento alguno para la realización del estudio.

\section{Conflicto de intereses}

Ningún conflicto de interés por declarar.

\section{Bibliografía}

1. Terry TL. Fibroblastic overgrowth of persistent tunica vasculosa lentis in infants born prematurely: II. Report of cases - clinical aspects. Trans Am Ophthalmol Soc. 1942;40:262-84.

2. Shah PK, Prabhu V, Karandikar SS, et al. Retinopathy of prematurity: past, present and future. World J Clin Pediatr. 2016;5:35-46.
3. Ramírez-Ortiz MA, Lara-Molina C, Villanueva-García D, et al. Retinopatía del prematuro: controversias en el uso de antiangiogénicos intraoculares. Bol Med Infant Mex 2013;70:344-50.

4. Flores-Nava G, Barrera-Vázquez C, Fuente-Torres M, et al. Retinopatía del prematuro. Determinación de algunos factores de riesgo. Bol Med Hosp Infant Mex. 2009;66:425-30.

5. Grupo de Trabajo Colaborativo Multicéntrico. Prevención de la ceguera en la infancia por ROP. Retinopatía del prematuro en servicios de neonatología de Argentina. Arch Argent Pediatr. 2006;104:69-74.

6. Gelman R. Diagnosis of plus disease in retinopathy of prematurity using retinal image multiscale analysis. IOVS. 2005;46(12).

7. Fierson WM; American Academy of Pediatrics Section on Ophthalmology; American Academy of Ophthalmology; American Association for Pediatric Ophthalmology and Strabismus; American Association of Certified Orthoptists. Screening examination of premature infants for retinopathy of prematurity. Pediatrics. 2013;131:189-95.

8. Chen J, Stahl A, Hellstrom A, et al. Current update on retinopathy of prematurity: screening and treatment. Curr Opin Pediatr. 2011;23:173-8.

9. McNamara JA, Tasman W, Brown GC, et al. Laser photocoagulation for stage 3+ retinopathy of prematurity. Ophthalmology. 1991;98:576-80.

10. Wu WC, Lien R, Liao PJ, et al. Serum levels of vascular endothelial growth factor and related factors after intravitreous bevacizumab injection for retinopathy of prematurity. JAMA Ophthalmol. 2015;133:391-7.

11. Shah PK, Narendran V, Kalpana N, et al. Anatomical and visual outcome of stages 4 and 5 retinopathy of prematurity. Eye (Lond). 2009;23:17680.

12. Mutlu FM, Sarici SU. Treatment of retinopathy of prematurity: a review of conventional and promising new therapeutic options. Int $\mathrm{J}$ Ophthalmol. 2013;6:228-36.

13. Kim MJ, Kim SJ, Yu YS. The risk for retinal detachment associated with hemorrhages pre- and postlaser treatment in retinopathy of prematurity. Retina. 2008;28:1451-7.

14. Oguchi C. Uber die Wirkung von Blutinjektionen in den Glaskorper nebst Bemwerkungen uber die Sogenannte Retinitis proliferans. Von Graefe's Archiv Fur Ophthalmlogie. 1913;84:446-520.

15. Cleary PE, Ryan SJ. Histology of wound, vitreous, and retina in experimental posterior penetrating eye injury in the Rhesus monkey. Am J Ophthalmol. 1979;88:221-31.

16. Hutcheson KA, Nguyen AT, Preslan MW, et al. Vitreous hemorrhage in patients with high-risk retinopathy of prematurity. Am J Ophthalmol. 2003;136:258-63. 